\title{
Power System State Estimation using a Direct Non-Iterative Method
}

\author{
Xinyu Tony Jiang a ${ }^{1}$ (Corresponding Author) and Joe H. Chow ${ }^{\text {a }}$ \\ ${ }^{\text {a }}$ Rensselaer Polytechnic Institute \\ 110 8th Street, Troy, NY, USA 12180 \\ Email: tjiang@quanta-technology.com and chowj@rpi.edu \\ Bruce Fardanesh $^{b}$, Deepak Maragal ${ }^{b}$, and George Stefopoulos ${ }^{b}$ \\ ${ }^{\mathrm{b}}$ New York Power Authority \\ 123 Main Street, White Plains, NY, USA 10601 \\ Email: Bruce.Fardanesh@nypa.gov, Deepak.Maragal@nypa.gov, and George.Stefopoulos@nypa.gov \\ Michael Razanousky ${ }^{c}$ \\ ${ }^{c}$ New York State Energy Research and Development Authority \\ 17 Columbia Circle, Albany, NY, USA 12203 \\ Email: mpr@nyserda.ny.gov
}

\begin{abstract}
This paper describes a new method for state estimation of a non-linear AC power system in a noniterative manner. This method is based on the Kipnis-Shamir relinearization technique that is used to solve over-defined sets of polynomial equations. The technique transforms the equations to a higher dimensional linear space which allows the states to be solved in a non-iterative manner. Given accurate measurements, this new state estimation method provides the same results as traditional iterative state estimation methods, and the proposed method does not require an initial guess of system states nor does it have issues with convergence.
\end{abstract}

\section{Keywords}

Non-Iterative Solution, Measurement-Based Solution, State Solution, State Estimation.

\footnotetext{
${ }^{1}$ Present affiliation:

Quanta Technology LLC

4020 Westchase Blvd., Suite 300

Raleigh, NC, USA 27607 


\section{Introduction}

Since its introduction in the late 1960s [1], power system state estimation has become an integral part of power system monitoring and operation. Because state estimation for alternating current (AC) power systems is a non-linear problem, traditionally state estimation has been solved using iterative methods such as the weighted least-squares with Gauss-Newton iterations [2], [3]. Iterative methods have worked well for the state estimator application, but these methods require an initial guess and may run into convergence issues if the initial guess is too far away from the actual system states [4].

This paper describes a new method for solving the state estimation problem of a non-linear AC power system in a non-iterative manner when given an adequate set of measurements [5]-[8]. There are other methods to solve the state estimation problem [9], [10] and the related power flow problem [11], [12] in a non-iterative manner by using linearized measurement functions and having some way to compensate for the linearized model. In contrast, the method proposed in this paper is based on the Kipnis-Shamir relinearization technique that is used to solve over-determined systems of polynomial equations [13]. In the proposed method, the measurement equations, which are the bus voltage magnitude, line power flow, and bus power injection equations, are formulated using rectangular representation of the bus voltages. With this formulation, the non-linear measurement equations become quadratic polynomials of the voltage variables. The method then uses two transformations to change the original system into a larger system to solve for the quadratic variables in a non-iterative manner. This new method provides the same results as the weighted least-squares method when given accurate measurements, and does not require an initial guess or have issues with solution convergence. 


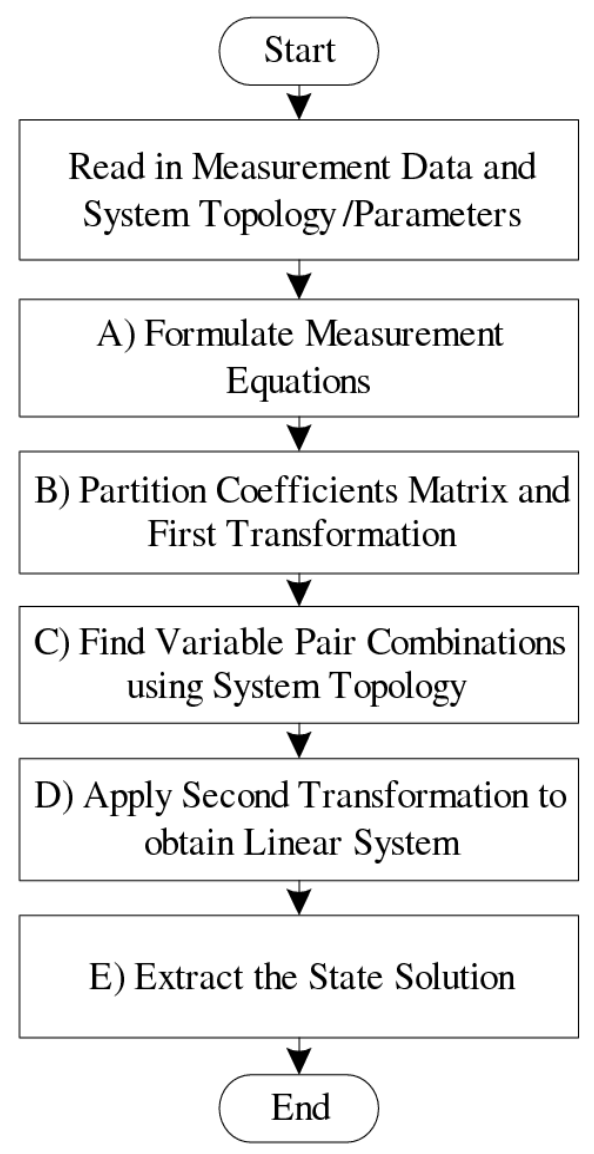

Figure 1 - Non-Iterative State Estimation Method Flow Chart

The main contributions of this paper are:

1. a comprehensive development of the algorithm needed for this non-iterative method, as shown in Figure 1, including techniques to reduce computation time, and

2. an assessment of this non-iterative method as compared to the conventional iterative state estimator solution.

The remainder of this paper is organized into five sections. Section II provides a description of the noniterative state estimation solution process. Section III presents a simple example to demonstrate the mechanics of the solution method. Section IV discusses the observability requirements of the proposed method. Section $V$ shows the performance test results of the proposed method, both for computation time and for solution accuracy when there are measurement errors. Finally, Section VI provides a summary and lists some future work to be done on this research topic.

\section{Non-Iterative State Estimation Method}

This section describes the non-iterative state estimation method. The inputs needed by the method are the system topology and parameter information and measurements from the system. 


\section{A. Formulating Measurement Equations}

In the method, the measurement equations are formulated using rectangular representation of the bus voltage phasors, i.e., $\bar{V}_{i}=V_{i R}+j V_{i I}$, such that the equations become quadratic polynomials in terms of these real and imaginary bus voltage components. If the transmission line parameters are expressed using the $\pi$-model shown in Figure 2 , then the measurement equations are:

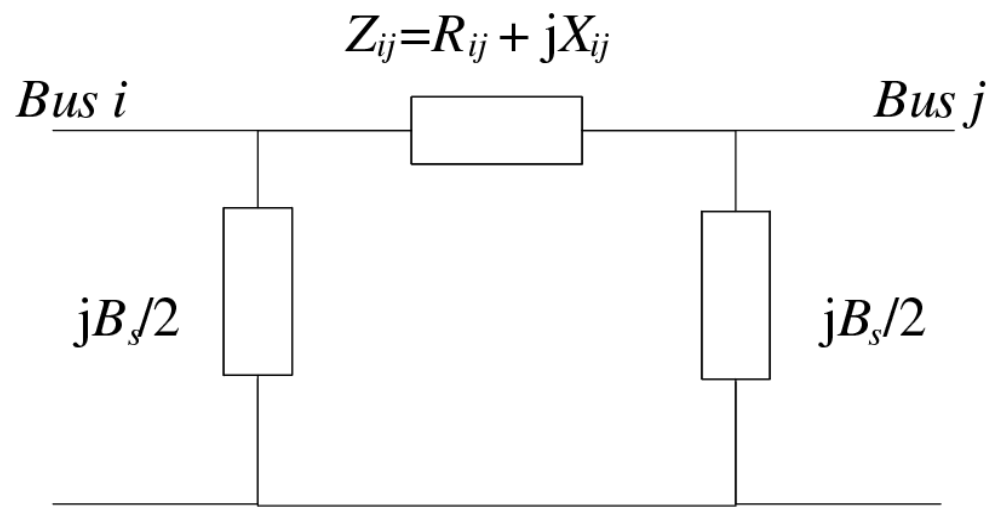

Figure 2 - Standard Transmission Line $\pi$-Model

a) Bus magnitude measurements

$$
V_{i M}^{2}=V_{i R}^{2}+V_{i I}^{2}
$$

where $V_{i M}$ is the magnitude of the voltage at Bus $i$.

b) Line active $(P)$ and reactive $(Q)$ power flow equations

$$
\begin{gathered}
P_{i j}=g_{i j}\left(V_{i R}^{2}+V_{i I}^{2}-V_{i R} V_{j R}-V_{i I} V_{j I}\right)+h_{i j}\left(V_{i I} V_{j R}-V_{i R} V_{j I}\right) \\
Q_{i j}=h_{i j}\left(V_{i R}^{2}+V_{i I}^{2}-V_{i R} V_{j R}-V_{i I} V_{j I}\right)+g_{i j}\left(V_{i R} V_{j I}-V_{i I} V_{j R}\right)-\frac{B_{s}}{2}\left(V_{i R}^{2}+V_{i I}^{2}\right) \\
g_{i j}=\frac{R_{i j}}{Z_{i j}^{2}}, h_{i j}=\frac{X_{i j}}{Z_{i j}^{2}}, Z_{i j}^{2}=R_{i j}^{2}+X_{i j}^{2}
\end{gathered}
$$

where Bus $i$ is the from bus and Bus $j$ is the to bus, and $R_{i j}, X_{i j}, B_{s}$ are the line resistance, reactance, and shunt susceptance, respectively.

c) Bus power injection equations are formulated by adding together all of the line flow equations that are going out of the bus plus the power flowing into any external shunt conductance $G$ or susceptance $B$ connected to the bus (e.g., a shunt capacitor or reactor on a bus):

$$
P=\sum P_{i j}+G\left(V_{i R}^{2}+V_{i I}^{2}\right)
$$




$$
Q=\sum Q_{i j}-B\left(V_{i R}^{2}+V_{i I}^{2}\right)
$$

where $j$ is the set of buses connected to Bus $i$.

Because these equations are linear with respect to the quadratic voltage terms $\left(V_{i R}^{2}, V_{i I}^{2}, V_{i R} V_{j R}\right.$, etc.), the equations can be put into the matrix form

$$
A_{\xi} \xi=C
$$

where $C$ is the vector of measurement values, $\xi$ is vector of quadratic voltage variables, and $A_{\xi}$ is the coefficient matrix for $\xi$. The vector $\xi$ consists of quadratic variables of the real and imaginary parts of the voltages, which are denoted by $x_{i} x_{j}$, where the indices $i$ and $j$ are not related to the bus numbers.

Line shunt conductance, non-unity transformer tap ratios and phase shifters can be added to the transmission line model. The resulting measurement equations will be more complex, but they are still quadratic polynomials of the voltage components.

\section{B. Partitioning $A_{\xi}$ and First Transformation}

A re-ordering of variables is performed and the system (4) is rearranged into

$$
\left[\begin{array}{ll}
A & B
\end{array}\right]\left[\begin{array}{l}
Y \\
Z
\end{array}\right]=C
$$

where $A$ contains the linearly independent columns of $A_{\xi}, B$ contains the remaining columns of $A_{\xi}, Y$ is the vector of elements of $\xi$ corresponding to $A$, and $Z$ is the vector of elements of $\xi$ corresponding to $B$. The quadratic variables $x_{i} x_{j}$ in $Y$ are renamed to $y_{1}, y_{2}, \ldots y_{N_{y}}$ in the order they appear, and $N_{y}$ is the total number of $Y$ variables. The quadratic variables $x_{i} x_{j}$ in $Z$ are renamed $z_{1}, z_{2}, \ldots z_{N_{z}}$ in the order they appear, and $N_{z}$ is the total number of $Z$ variables.

In addition, all quadratic variables containing the reference bus imaginary component and their corresponding columns in the matrices are eliminated from the system because the reference bus imaginary component is set to zero. In a software implementation, the partitioning process can be reliably performed by using QR decomposition [6] on $A_{\xi}$.

With the rearranged system, the $Y$ variables can now be expressed in terms of the $Z$ variables and the measurement values $C$ as

$$
\begin{gathered}
Y=d+D Z \\
d=\left(A^{T} A\right)^{-1} A^{T} C, \quad D=-\left(A^{T} A\right)^{-1} A^{T} B
\end{gathered}
$$

The $A$ matrix is a tall matrix if there are redundant measurements. 
The system is transformed into

$$
\left[\begin{array}{c}
y_{1} \\
\vdots \\
y_{N_{Y}} \\
z_{1} \\
\vdots \\
z_{N_{Z}}
\end{array}\right]=E \cdot\left[\begin{array}{c}
1 \\
z_{1} \\
\vdots \\
z_{N_{Z}}
\end{array}\right], \quad E=\left[\begin{array}{cc}
d & D \\
0 & \\
\vdots & I \\
0 &
\end{array}\right]
$$

where the new matrix $E$ is formed by combining $d$ and $D$ and then appending a zero column and an identity matrix $I$ of dimension $N_{z}$.

In this formulation, $d$ is the only part of $E$ that depends on the measurement values $C$. It should be noted that $A$ and $B$ are determined by the system topology and parameters, and thus $D$ and by extension the identity matrix lying below it in the $E$ matrix are dependent only on the topology of the system and not on the measurement values. This means that as long as the system topology remains unchanged, only $d$ has to be recomputed when a new batch of measurements arrive and the remaining parts of $E$ can be taken from previous calculations. This provides significant computational savings for the method.

\section{Finding Variable Product Pair Combinations}

In this step, unique variable product pair combinations of the quadratic variables are formed. A valid product pair satisfies

$$
s_{i j} s_{p q}=\left(x_{i} x_{j}\right)\left(x_{p} x_{q}\right)=\left(x_{i} x_{p}\right)\left(x_{j} x_{q}\right)=s_{i p} s_{j q}
$$

where $i, j, p, q=1, \ldots, n_{\max }$, and $n_{\max }$ corresponds to the total number of $x$ (bus voltage component) variables.

These product pair relationships are used to impose additional constraints on the system so the correct solution can be obtained. For a product pair to be valid, the terms $s_{i j}, s_{p q}, s_{i p}$, and $s_{j q}$ must exist among the set of $Y$ and $Z$ quadratic variables for the system, and $s_{i j}$ and $s_{p q}$ cannot be the same pair as $s_{i p}$ and $s_{j q} \cdot$

It is recognized that only certain quadratic variable pair equations (8) are valid. In the Appendix, we show the specific network structures that will generate valid product pairs. Searching for these simple structures in a system and generating the possible product pairs to check is considerably faster than a brute-force exhaustive search over all combinations of quadratic variables.

An additional observation is that the set of valid product pair combinations depends on the system topology and available measurements but not on the measurement values themselves. This means that as long as the system topology and set of available measurements are unchanged, the set of valid 
combinations can be reused from previous calculations, and this can provide savings in computation time.

\section{Second Transformation into Linear System}

For each valid product pair (8), one equation in the form

$$
s_{i j} s_{p q}-s_{i p} s_{j q}=s_{l_{1}} s_{l_{2}}-s_{l_{3}} s_{l_{4}}=0
$$

can be generated. Due to the representation of the $s(Y$ and $Z$ ) quadratic variables in (7), the product of two $s$ variables will result in a sum composed of a scalar with linear and quadratic terms of the $Z$ variables. A way to calculate the coefficients is to multiply rows of the $E$ matrix corresponding to the quadratic variables in question as

$$
s_{i j} s_{p q}-s_{i p} s_{j q}=s_{l_{1}} s_{l_{2}}-s_{l_{3}} s_{l_{4}} \rightarrow E_{l_{1}}^{T} \cdot E_{l_{2}}-E_{l_{3}}^{T} \cdot E_{l_{4}}
$$

where $E_{l_{1}}$ is the $l_{1}$-th row of the $E$ matrix. The result of each operation in (10) results in a square matrix of dimension $N_{z}+1$. Due to the relationship shown in (7), the terms in the square matrix are coefficients for the $Z$ variables or quadratic combinations of the $Z$ variables:

$$
\left[\begin{array}{ccccc}
1 & z_{1} & z_{2} & \cdots & z_{N_{Z}} \\
z_{1} & z_{1}^{2} & z_{1} z_{2} & \cdots & z_{1} z_{N_{Z}} \\
z_{2} & z_{1} z_{2} & z_{2}^{2} & \cdots & z_{2} z_{N_{Z}} \\
\vdots & \vdots & \vdots & \ddots & \vdots \\
z_{N_{Z}} & z_{1} z_{N_{Z}} & z_{2} z_{N_{Z}} & \cdots & z_{N_{Z}}^{2}
\end{array}\right]
$$

The 1 in the top left corner of (11) is a representation of the measurement values and is not related to any of the $Z$ variables.

The rows of the each square matrix are concatenated from start to end to form a long row and the long rows are then organized into the matrix $A_{t}^{\prime}$, such that the resulting system of equations becomes 


$$
A_{t}^{\prime}\left[\begin{array}{c}
1 \\
z_{1} \\
\vdots \\
z_{N_{Z}} \\
z_{1} \\
z_{1}^{2} \\
z_{1} z_{2} \\
\vdots \\
z_{1} z_{N_{Z}} \\
\vdots \\
z_{N_{Z}} \\
z_{1} z_{N_{Z}} \\
\vdots \\
z_{N_{Z}}^{2}
\end{array}\right]=\left[\begin{array}{c}
0 \\
\vdots \\
0
\end{array}\right]
$$

The number of rows in $A_{t}^{\prime}$ depends upon the number of variable product pairs that satisfy (8), and the number of columns in $A_{t}^{\prime}$ is $\left(N_{z}+1\right)^{2}$.

As seen in (12), some of the columns of $A_{t}^{\prime}$ represent the same $Z$ or quadratic $Z$ variables. Therefore these columns have to be combined by adding them together. The newly compacted $A_{t}^{\prime}$ matrix is then partitioned as

$$
\text { compacted } A_{t}^{\prime}=\left[\begin{array}{ll}
-k & A_{t}
\end{array}\right]
$$

where $-k$ is the first column of $A_{t}^{\prime}$, and $A_{t}$ contains the remaining columns.

The system can now be reformulated in the $t$ variables as

$$
\begin{aligned}
& A_{t} t=k \\
& t=\left[\begin{array}{llllllllllllll}
z_{1} & z_{2} & \cdots & z_{N_{Z}} & z_{1}^{2} & z_{1} z_{2} & \cdots & z_{1} z_{N_{Z}} z_{2}^{2} & z_{2} z_{3} & z_{2} z_{4} & \cdots & z_{N_{Z}-1} z_{N_{Z}} & z_{N_{Z}}^{2}
\end{array}\right]^{T}
\end{aligned}
$$

The number of $t$ variables is

$$
N_{t}=N_{z}+\frac{N_{z}\left(N_{z}+1\right)}{2}
$$

Equation (14) is now a linear system of the $t$ variables and therefore can be solved directly. An important issue with the transformation is that the process can take considerable amount of computation time for large power systems. As the number of $t$ variables is proportional to the square of the number of $Z$ variables which is linearly related to the system size, a system with thousands of buses will result in $A_{t}$ having millions of columns. However, $A_{t}$ is very sparse, and thus solving this system with sparse matrix techniques can still be done efficiently. The problem is that generating the rows of $A_{t}$ can be time-consuming. From the formulation, one can see that the number of columns of $A_{t}$ and $A_{t}^{\prime}$ is 
dependent on the number of $Z$ variables, and the number of $Z$ variables is a function of the system size, topology, and measurement set. More $Z$ variables translate into a larger $A_{t}$ matrix, which means that the calculation will take longer to solve.

Generating the rows of $A_{t}$ with (10) and flattening the resulting square matrices into the rows is unavoidable when the non-iterative method is invoked for the first time on a system. However, the effort to generate $A_{t}$ can be greatly reduced for subsequent runs on the same system with the same set of measurements. To illustrate, partition $A_{t}$ and $\mathrm{t}$ as

$$
\begin{aligned}
& A_{t} t=\left[\begin{array}{ll}
A_{t Z} & A_{t Z_{2}}
\end{array}\right]\left[\begin{array}{l}
Z \\
Z_{2}
\end{array}\right] \\
& Z=\left[\begin{array}{llll}
z_{1} & Z_{2} & \cdots & z_{N_{Z}}
\end{array}\right]^{T} \\
& z_{2}=\left[\begin{array}{lllllllll}
z_{1}^{2} & z_{1} z_{2} & \cdots & z_{1 z_{N_{Z}}} z_{2}^{2} & z_{2} z_{3} & \cdots & z_{N_{Z}-1} z_{N_{Z}} & z_{N_{Z}}^{2}
\end{array}\right]^{T}
\end{aligned}
$$

where $A_{t Z}$ contains the columns of $A_{t}$ corresponding to the linear $Z$ variables, and $A_{t Z_{2}}$ contains the columns corresponding to the quadratic combinations $Z$ variables (which will be referred to as the $Z_{2}$ terms). It is important to note that the $A_{t Z_{2}}$ portion is obtained from the multiplications of terms in the columns of $E$ that are not dependent on the measurement values. Because the product pair combinations used to generate $A_{t}$ are not dependent on the measurement values, the entirety of $A_{t z_{2}}$ is not dependent on the measurement values. Only the $A_{t z}$ portion needs to be recomputed when the measurement values are updated. This translates into significant savings in computation time, as $A_{t Z}$ only has $N_{z}$ columns compared to the $N_{z}+\frac{N_{Z}\left(N_{z}+1\right)}{2}$ columns of the entire $A_{t}$.

The ultimate goal of transforming the system into (14) is to solve for the values of the $Z$ variables. Due to its construction, the columns of $A_{t Z_{2}}$ portion may be linearly dependent with other columns in $A_{t Z_{2}}$. The peculiar structure of $A_{t}$ means that while the individual values of the $Z$ variables associated with the $A_{t Z}$ columns may be solved uniquely, the individual values of the $Z_{2}$ variables associated with $A_{t Z_{2}}$ may not be solved uniquely. However, only a unique solution to the $Z$ variables is needed, and thus it does not matter if the $Z_{2}$ variables cannot be solved uniquely.

The two necessary conditions for $A_{t}$ to ensure a unique solution to the $Z$ variables are

$$
\begin{aligned}
& \operatorname{rank}\left(A_{t Z}\right)=\text { number of columns of } A_{t Z} \\
& \operatorname{rank}\left(A_{t}\right)=\operatorname{rank}\left(A_{t Z}\right)+\operatorname{rank}\left(A_{t Z_{2}}\right)
\end{aligned}
$$

If (18) is not met, then the solution to some of the $Z$ variables may not be found correctly, indicating that there are not sufficient measurements to solve for all of the quadratic voltage variables in the system. The requirements for the method to solve for all system states, also known as observability requirements, are discussed in Section IV. 


\section{E. Extracting the State Solution}

After obtaining the solution of the $t$ variables, the values of the $Z$ variables can be extracted directly from $t$ because the first $N_{Z}$ entries of $t$ are the $Z$ variables. The values of the $Y$ variables can be solved from the $Z$ variables with (6).

Because the indices of individual bus voltage components that made up each $Y$ and $Z$ variable were recorded, the values of the voltage components can be extracted from the $Y$ and $Z$ variables. The voltage components can either be extracted sequentially by taking square roots and doing division, or by using an iterative least-squares method. For the sequential approach, in order to solve for the signs of the voltage components correctly, one has to start at the buses where the signs are known (the reference bus and perhaps other designated buses) and use the non-square quadratic terms to determine the signs of adjacent buses. This process is repeated until the signs to all voltage components have been determined.

\section{F. Choosing the Reference Bus}

The location of the reference bus has an effect on the solution process. Setting the reference to a bus would eliminate all quadratic variables containing the imaginary part of that bus voltage, and thus different reference locations change the set of quadratic variables in the system. This has an effect on the error rejection capability of the method when there is noise in the measurements. Testing has shown that setting the reference to a bus with many connections results in lower solution error. It is also important to set the reference to a bus with the accurate measurements.

If there are synchrophasor measurements in the system, the reference should be set to a bus with voltage phasor measurements. This is because the phase angle of the bus voltage is already measured at those locations, so it is natural to choose one of those locations for the reference.

\section{A Simple Example}

A simple two-bus example shown in Figure 3 is used to demonstrate the mechanics of the non-iterative calculation method. The quantities to be solved are the voltage phasors at each bus.

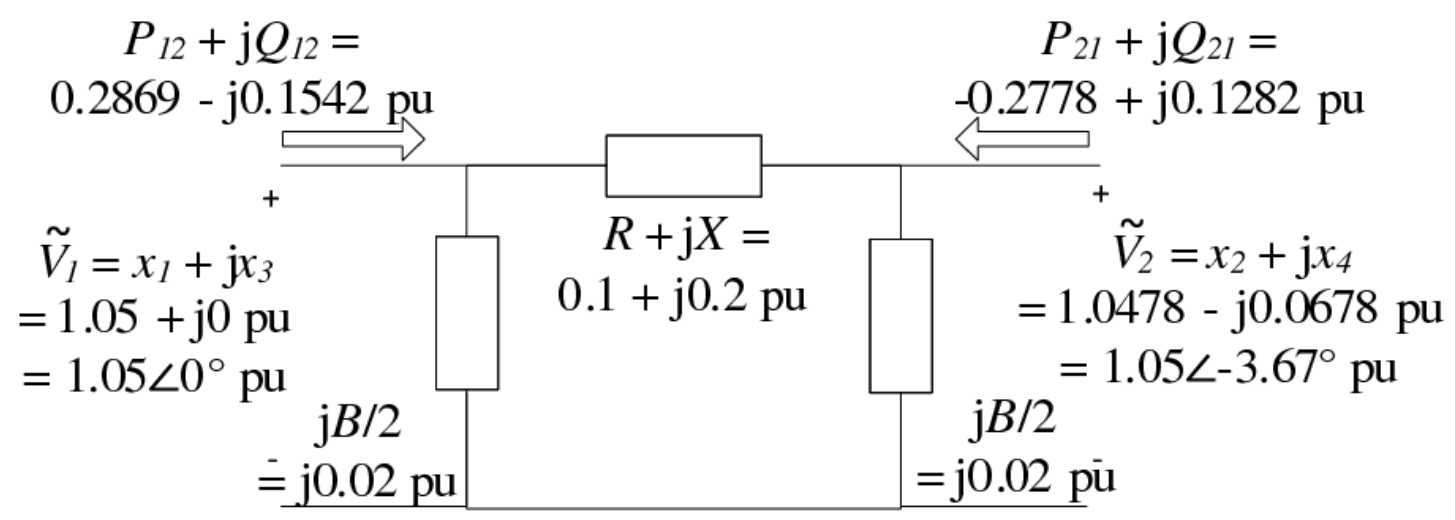

Figure 3 - Example Two-bus, One-Line System 
For this example, it is assumed that the voltage magnitudes at both buses are known, and so is active $(P)$ and reactive $(Q)$ power flow measurements from Bus 1 to Bus 2, resulting in a total of four measurement equations. Bus 1 is set as the reference, and thus its imaginary component becomes 0 , leaving three unknown voltage components to be solved from four measurement equations. To simplify the notation, the real and imaginary voltage components of Bus 1 are designated as $x_{1}$ and $x_{3}$, respectively, and the real and imaginary voltage components of Bus 2 are designated as $x_{2}$ and $x_{4}$, respectively.

The four measurement equations in the form of (2) are put into matrix form as

$$
\left[\begin{array}{cccccccc}
1 & 1 & 0 & 0 & 0 & 0 & 0 & 0 \\
0 & 0 & 1 & 1 & 0 & 0 & 0 & 0 \\
2 & 2 & 0 & 0 & -2 & -2 & -4 & 4 \\
3.98 & 3.98 & 0 & 0 & -4 & -4 & 2 & -2
\end{array}\right]\left[\begin{array}{c}
x_{1} x_{1} \\
x_{3} x_{3} \\
x_{2} x_{2} \\
x_{4} x_{4} \\
x_{1} x_{2} \\
x_{3} x_{4} \\
x_{1} x_{4} \\
x_{2} x_{3}
\end{array}\right]=\left[\begin{array}{c}
1.1025 \\
1.1025 \\
0.2869 \\
-0.1542
\end{array}\right]
$$

The measurement equations produce eight quadratic voltage variables. However, since $x_{3}$ is zero, three of the quadratic terms and their associated columns are eliminated from the system, leaving only five quadratic variables. The system is now partitioned into the form of (5) and the first variable change is performed:

$$
\begin{gathered}
{\left[\begin{array}{cccc}
1 & 0 & 0 & 0 \\
0 & 1 & 0 & 0 \\
2 & 0 & -2 & -4 \\
3.98 & 0 & -4 & 2
\end{array}\right]\left[\begin{array}{l}
y_{1} \\
y_{2} \\
y_{3} \\
y_{4}
\end{array}\right]+\left[\begin{array}{l}
0 \\
1 \\
0 \\
0
\end{array}\right] \mathrm{z}_{1}=\left[\begin{array}{c}
1.1025 \\
1.1025 \\
0.2869 \\
-0.1542
\end{array}\right]} \\
{\left[\begin{array}{l}
y_{1} \\
y_{2} \\
y_{3} \\
y_{4} \\
z_{1}
\end{array}\right]=\left[\begin{array}{l}
x_{1} x_{1} \\
x_{2} x_{2} \\
x_{1} x_{2} \\
x_{1} x_{4} \\
x_{4} x_{4}
\end{array}\right]}
\end{gathered}
$$

The resulting matrix $E$ from (7) is calculated as

$$
E=\left[\begin{array}{cc}
1.1025 & 0 \\
1.1025 & -1 \\
1.1002 & 0 \\
-0.0706 & 0 \\
0 & 1
\end{array}\right]
$$

The variable product pair combinations that satisfy (8) are identified. The two combinations found are 


$$
\begin{aligned}
& y_{1} y_{2}=\left(x_{1} x_{1}\right)\left(x_{2} x_{2}\right)=\left(x_{1} x_{2}\right)\left(x_{1} x_{2}\right)=y_{3} y_{3} \\
& y_{1} z_{1}=\left(x_{1} x_{1}\right)\left(x_{4} x_{4}\right)=\left(x_{1} x_{4}\right)\left(x_{1} x_{4}\right)=y_{4} y_{4}
\end{aligned}
$$

The second transformation is applied, and thus the $A_{t}^{\prime}$ matrix is generated from the rows of $E$ with (22)(23) and operation (10). The system is now transformed into the form of (12) as

$$
A_{t}^{\prime}\left[\begin{array}{l}
1 \\
z_{1} \\
z_{1} \\
z_{1}^{2}
\end{array}\right]=\left[\begin{array}{l}
0 \\
0
\end{array}\right], \quad A_{t}^{\prime}=\left[\begin{array}{cccc}
0.005 & 0 & -1.1025 & 0 \\
-0.005 & 0 & 1.1025 & 0
\end{array}\right]
$$

The $A_{t}^{\prime}$ matrix is compacted and the system is rearranged into the form in (14) as

$$
A_{t} t=\left[\begin{array}{cc}
-1.1025 & 0 \\
1.1025 & 0
\end{array}\right]\left[\begin{array}{l}
t_{1} \\
t_{2}
\end{array}\right]=\left[\begin{array}{c}
-0.005 \\
0.005
\end{array}\right], \quad\left[\begin{array}{l}
t_{1} \\
t_{2}
\end{array}\right]=\left[\begin{array}{l}
z_{1} \\
z_{1}^{2}
\end{array}\right]
$$

Only $t_{1}$ can be solved, but that is all that is needed because there is only one $Z$ variable, and therefore

$$
t_{1}=z_{1}=x_{4} x_{4}=0.0045
$$

Using (6), the $y$ variables are solved as

$$
\left[\begin{array}{l}
y_{1} \\
y_{2} \\
y_{3} \\
y_{4}
\end{array}\right]=\left[\begin{array}{l}
x_{1} x_{1} \\
x_{2} x_{2} \\
x_{1} x_{2} \\
x_{1} x_{4}
\end{array}\right]=\left[\begin{array}{cc}
1.1025 & 0 \\
1.1025 & -1 \\
1.1002 & 0 \\
-0.0706 & 0
\end{array}\right]\left[\begin{array}{l}
1 \\
z_{1}
\end{array}\right]=\left[\begin{array}{c}
1.1025 \\
1.0980 \\
1.1002 \\
-0.0706
\end{array}\right]
$$

The magnitudes of the individual voltage component can be solved by taking the square root of the squared quadratic terms of the real components and solving the other components by division. To determine the signs, it is assumed that the real component of reference voltage is positive:

$$
\begin{gathered}
x_{1}=\sqrt{1.1025}=1.05, \quad x_{2}=\sqrt{1.0980}=1.0478, \\
x_{3}=0, \quad x_{4}=-\left(\frac{0.0706}{1.05}\right)=-0.0672
\end{gathered}
$$

\section{Observability Requirements}

Whether a system is solvable or observable (in state estimation terminology) using the non-iterative calculation method depends on the number of measurements and the network connectivity and topology. Due to the nature of the proposed method, the number of measurements should be larger 
than the number of unknowns to yield a solution. When a solution of the linear set of equations in the $t$ variables is not possible, this may indicate a lack of sufficient measurements.

In the two-bus example system shown in Section III, there are three unknown voltage components due to the reference bus imaginary component being set to zero. The observability of the system using the non-iterative state estimation method is outlined Table I. Not surprisingly, the system is observable with any combination of four or more measurements as there are only three variables to be solved. There is a special three-measurement case in the middle row of Table I that is solvable because it results in a linear system for the voltage variables on bus 2 . Any other configuration with three or fewer measurements cannot be solved correctly with the non-iterative method.

Table I - Two-Bus Example System Observability

\begin{tabular}{|l|l|l|l|}
\hline Measurements Used & $\begin{array}{l}\text { Number of } \\
\text { Measurements }\end{array}$ & Reference Bus & Solvable? \\
\hline $\begin{array}{l}4 \text { or more } \\
\text { measurements }\end{array}$ & $\geq 4$ & 1 or 2 & Yes \\
\hline$V_{1}, P_{12} \& Q_{12}$ & 3 & 1 & Yes \\
\hline $\begin{array}{l}\text { Any other combination } \\
\text { of } 3 \text { or fewer } \\
\text { measurements }\end{array}$ & $\leq 3$ & 1 or 2 & No, incorrect solution \\
\hline
\end{tabular}

Expanding upon the results in Table I, it can be reasoned that the minimum set of measurements to ensure observability would be to have four measurements covering each branch (two-bus connection).

This statement is made under the assumption that the measurement types are limited to bus voltage magnitudes, line power flows and bus power injections, and all line parameters are known. Voltage magnitudes measurements are good to have because these measurements can cover for more than one branch, but line flow and bus injection measurements are needed to relate the values of adjacent buses. The requirement of four measurements per branch means that each branch should have at least two line flow or bus injection measurements ( $P$ and $Q$ are considered to be separate), because only two voltage magnitudes are available. Having more than the minimum number of measurements would provide redundancy to the measurement set, and also improve the solution error performance as explained in Section V.B.

In real power systems, the utility companies typically have measurements in the most important portions of its state estimation system model. However, the measurements usually do not cover the entire model. To fill in the gaps, pseudo-measurements based on historical load profiles are inserted so the state estimator can give a solution for the model. The non-iterative method proposed in this paper can also make use of these pseudo-measurements.

Compared to the traditional weighted least-squares, the non-iterative method requires more measurements for observability. Looking at the cases in Table I, the traditional method can solve any cases with 3 or more measurements. This is because the non-iterative method tries to calculate a 
solution, while the traditional method iterates towards the solution. The need for a higher number of measurements is a potential drawback for the non-iterative method.

\section{Performance Test Results}

This section presents performance test results of the non-iterative state estimation method. The new method has been implemented using MATLAB [14] and tested on systems up to 1446 buses. In all cases, the method produced solutions that agree with solutions from the classical weighted least-squares estimator with the same set of measurements. In these tests, the measurement values were emulated via traditional power flow solutions. The measurements supplied in the tests were the voltage magnitude measurements at all buses and line power flow on all lines in one direction. In the tests, the execution times of the MATLAB program takes to solve the test systems are measured using MATLAB's built-in stopwatch functions. The computer used to run the MATLAB program has an Intel Core i5 M 540 processor and 4 gigabytes of memory. All computation times listed here are approximate averages over several runs. 
Table II shows the computation times of the non-iterative method for an 1189-bus, 1508-line system and a 1446-bus, 2856-line system. Two non-iterative method total times are listed for each system: the first total time is for an initial run where the topology and system parameters have to be processed and the variable product pairs have to be found; the second total time is for a subsequent run where the system information has already been processed and only the measurement values have changed. The computation times for the steps of the initial run are also listed in the table.

In addition, the solutions times for an iterative weighted least-squares state estimator programmed in MATLAB are listed in the last row of the tables for comparison. 
Table II - Non-Iterative State Estimation Computation Times

\begin{tabular}{|l|l|l|l|l|}
\hline Steps & 1189-bus 1 thread & $\begin{array}{l}\text { 1189-bus 2 } \\
\text { threads }\end{array}$ & 1446-bus 1 thread & $\begin{array}{l}\text { 1446-bus } 2 \\
\text { threads }\end{array}$ \\
\hline $\begin{array}{l}\text { A. Formulating } \\
\text { equations and } \\
\text { partitioning the } \\
\text { system }\end{array}$ & 1.9 seconds & 1.9 seconds & 4 seconds & 4 seconds \\
\hline $\begin{array}{l}\text { B. First } \\
\text { transformation }\end{array}$ & 0.6 seconds & 0.6 seconds & 1.2 seconds & 1.2 seconds \\
\hline $\begin{array}{l}\text { C. Generating } \\
\text { possible variable } \\
\text { pair combinations }\end{array}$ & 0.5 seconds & 0.5 seconds & 1.2 seconds & 1.2 seconds \\
\hline $\begin{array}{l}\text { D. Second } \\
\text { transformation }\end{array}$ & 2.3 seconds & 1.5 seconds & 7.0 seconds & 5 seconds \\
\hline $\begin{array}{l}\text { E. Solving the } \\
\text { linear system }\end{array}$ & 1.4 seconds & 1.4 seconds & 2.9 seconds & 2.9 seconds \\
\hline $\begin{array}{l}\text { F. Extracting the } \\
\text { solution }\end{array}$ & 0.1 seconds & 0.1 seconds & 0.1 seconds & 0.1 seconds \\
\hline Total, initial run & 6.8 seconds & 6.0 seconds & 16.4 seconds & 14.4 seconds \\
\hline $\begin{array}{l}\text { Total, subsequent } \\
\text { run }\end{array}$ & 1.2 seconds & 0.8 seconds & 5 seconds & 2.8 seconds \\
\hline $\begin{array}{l}\text { Total, weighted } \\
\text { least-squares }\end{array}$ & 1.1 seconds & & 3 seconds & \\
\hline
\end{tabular}


Table II shows that the initial run of the non-iterative method is considerably slower than the classical iterative least-squares method. In the initial run, the step that takes the most time is Step $D$, where the $A_{t}$ matrix from (14) is generated. Step $\mathrm{D}$ takes considerable time because the dimensions of $A_{t}$ become very large for larger systems.

While the initial run is slower compared to the classical method, the computation times of subsequent runs using the non-iterative method are much more competitive. In a subsequent run, Steps $A$ and $C$ in 
Table II are unnecessary. The computation effort is also drastically reduced for Steps B and D because only a small portion of the $E$ and $A_{t}$ matrices need to be recalculated when only the measurement values change, and all of this translate into significant speed-ups.

\section{A. Improving Performance with Parallel Computing}

The computation time of the non-iterative method can be further improved by using parallel computing techniques. Most modern computers feature multi-core processors, and only software that can execute on multiple processing threads can take advantage of the computing power offered by multiple cores. In state estimation, most prior literature introduces parallel computing by breaking down a larger system into smaller subsystems and running state estimations on the subsystems in parallel [15], [16]. After the subsystems are solved, a state estimation is run on the higher level to ensure that the solutions at the subsystem boundaries are consistent.

Such an approach, known as a hierarchical estimator, is equally applicable to the non-iterative method as it is to the weighted least-squares method or any related iterative methods. However, the noniterative method can also benefit from introducing parallel processing into the algorithm itself. Within the non-iterative solution process, parallel processing can be readily added to Step D. In Step D, each row of $A_{t}^{\prime} / A_{t}$ is generated independently of other rows, and so the task can be split between multiple cores. Parallel processing is applicable to both the initial run and subsequent runs, as subsequent runs have to recalculate a portion of the $A_{t}$ matrix.

Parallel processing was added to the MATLAB program by the parfor function within MATLAB's Parallel Computing Toolbox [17]. 
Table II shows the computation times for the 1189-bus and 1446-bus systems with one processing thread and two processing threads. 
Table Il shows that using two processing threads brings improvements in computation time for both the initial run and the subsequent run. The initial run contains many calculations that are not parallelized and so the improvement is limited. In contrast, the subsequent run consists mainly of a modified version of the Step D calculation, and therefore adding parallel processing improves the subsequent run computation time significantly. Many modern computers feature four or more processor cores, and the computation time can be further decreased by running more parallel threads. The 
Table II results do not show a parallelized running time for the iterative state estimator because the iterative state estimation algorithm is not easy to parallelize. The ability of the new non-iterative method to take advantage of parallel processing may be an advantage over the iterative methods.

\section{B. Effect of Measurement Noise/Error}

The effect of measurement noise/error on the non-iterative calculation method was investigated using MATLAB, and the performance of the non-iterative method and the traditional weighted least-squares method are compared. The solution error of the non-iterative method is affected by the method used to extract the voltage components from the solutions of the quadratic voltage variables, and therefore two extraction methods are presented in the results. The first is a sequential approach where the magnitudes are first solved using any available square terms and then the remaining terms are solved using division and the non-square terms. The second approach uses an iterative least-squares formulation to solve for the individual voltage components.

The solution error performance was evaluated on the 1189-bus test system by adding different levels of normally distributed noise to all measurements. The standard deviation of the noise is defined as a percentage of the ideal measurement values. The choice of 1-3\% standard deviation is for illustrative purposes only. In real systems, it is realistic to expect the error in voltage measurements to be generally less than $1 \%$, whereas current measurement errors may be higher.

For all tests, the measurement set includes all bus voltage magnitudes. The line power flow measurements on all lines are supplied in either one or both directions, and the reason for having two separate cases is to illustrate the impact of the number of measurements on the solution error. The reference bus is set to a bus with many connections.

The test results are shown in Table III. Each cell in the table displays two numbers: the first number is the average voltage vector error (per bus), and the second is the maximum voltage vector error.

If the voltage phasor solution at a bus with noisy measurements is designated as $V_{R}+j V_{I}$ and the ideal solution is designated as $V_{R o}+j V_{I o}$, then the vector error is calculated as

$$
V_{E}=\sqrt{\left(V_{R}-V_{R o}\right)^{2}+\left(V_{I}-V_{I o}\right)^{2}}
$$

The results in Table III show that the classical weighted least-squares method has smaller solution errors than the non-iterative method. 
Table III - Voltage Vector Error With Normally-Distributed Noise, 1189-bus (Average of 10 Runs)

\begin{tabular}{|c|c|c|c|}
\hline Standard Deviation & $1 \%(p u)$ & $2 \%(p u)$ & $3 \%(p u)$ \\
\hline $\begin{array}{l}\text { Weighted least- } \\
\text { squares, Line flows } \\
\text { both directions }\end{array}$ & $\begin{array}{l}0.0014 \\
0.0182\end{array}$ & $\begin{array}{l}0.0028 \\
0.0330\end{array}$ & $\begin{array}{l}0.0044 \\
0.0661\end{array}$ \\
\hline $\begin{array}{l}\text { Weighted least- } \\
\text { squares, Line flows one } \\
\text { direction }\end{array}$ & $\begin{array}{l}0.0018 \\
0.0226\end{array}$ & $\begin{array}{l}0.0057 \\
0.0380\end{array}$ & $\begin{array}{l}0.0053 \\
0.0777\end{array}$ \\
\hline $\begin{array}{l}\text { Non-Iterative Method, } \\
\text { Sequential extraction, } \\
\text { Line flows both } \\
\text { directions }\end{array}$ & $\begin{array}{l}0.0030 \\
0.1516\end{array}$ & $\begin{array}{l}0.0053 \\
0.1239\end{array}$ & $\begin{array}{l}0.0080 \\
0.1551\end{array}$ \\
\hline $\begin{array}{l}\text { Non-Iterative Method, } \\
\text { Sequential extraction, } \\
\text { Line flows one } \\
\text { direction }\end{array}$ & $\begin{array}{l}0.0422 \\
0.4269\end{array}$ & $\begin{array}{l}0.0699 \\
0.4948\end{array}$ & $\begin{array}{l}0.0906 \\
0.6759\end{array}$ \\
\hline $\begin{array}{l}\text { Non-Iterative Method, } \\
\text { Least-squares } \\
\text { extraction, Line flows } \\
\text { both directions }\end{array}$ & \begin{tabular}{|l|}
0.0020 \\
0.0257
\end{tabular} & $\begin{array}{l}0.0040 \\
0.0464\end{array}$ & $\begin{array}{l}0.0064 \\
0.0815\end{array}$ \\
\hline $\begin{array}{l}\text { Non-Iterative Method, } \\
\text { Least-squares } \\
\text { extraction, Line flows } \\
\text { both directions }\end{array}$ & $\begin{array}{l}0.0112 \\
0.0467\end{array}$ & $\begin{array}{l}0.0231 \\
0.0942\end{array}$ & $\begin{array}{l}0.0362 \\
0.1530\end{array}$ \\
\hline
\end{tabular}

To look deeper into this result, observe that the solution error of the non-iterative method is smaller when there are line flows in both directions than in one direction. This result can be explained by the fact that when there are line flow measurements in both directions on all lines in the system, the $A$ matrix from (5) will be a tall matrix, and thus the operation (6) becomes a least-squares solution. This least-squares component gives the non-iterative method some noise rejection capability. If the line flows are only in one direction, then $A$ is square and (6) has no noise rejection capability.

For the non-iterative method, the least-squares approach to extract the voltage component solution results in smaller solution errors than the simple sequential approach. The larger error of the sequential approach is because the value of each voltage component is calculated from only one quadratic variable. Since the accuracy of the quadratic variable solution is not known beforehand, the sequential method may pick quadratic variables that have large errors, which results in large errors in the voltage vector solution. The least-squares approach takes into account the value of all quadratic variables, thus minimizing the solution error.

The iterative extraction process in the last step is less likely to have convergence issues than the traditional iterative state estimation methods, as the equations are much simpler. Convergence 
problems tend to occur when the starting guess is too far away from the actual solution. A way to get a good starting guess for the iterative extraction is to run the sequential extraction, which takes a negligible amount of processing time, and use that solution as the starting guess.

Work is currently being done

\section{Observations}

Based on the analysis done in this section and Section IV, the following observations can be made:

1. The non-iterative method requires more measurements than the conventional iterative SE solution, which is obvious from Table I. In some sense this is to be expected as more information is needed to generate enough equations for the non-iterative method to provide a solution.

2. As a consequence of the first observation, to achieve the same level of least-squares errors, the non-iterative method requires more measurements than the iterative method. To illustrate, in the $2 \%$ column in Table III, the LS errors in the iterative method with line flows in one direction are similar in magnitude to the non-iterative method with LS extraction using line flows in both directions.

\section{Summary and Future Work}

A contribution of this paper is the development the algorithmic details of a non-iterative method to solve the power system state estimation problem. The method offers the advantages of being independent of the initial guess values required by iterative methods and thus not having solution convergence issues. In terms of computation time, the non-iterative method is competitive with traditional iterative methods when the network topology setting is reused and parallel computing is enabled. A second contribution is the demonstration of the limitations of the non-iterative method. The proposed method requires more measurements than traditional iterative methods to develop enough equations such that a solution can be obtained. As a result, given the same noisy measurements, the non-iterative method will result in a higher solution error. An iterative step at the end of the solution process can be used to lower the solution error, although adding iterations is not a desired solution. Work is currently being done on non-iterative ways to lower the solution error.

A potential application of the non-iterative method is its role in a hierarchical state estimator in which the high-voltage level state estimation is computed separately using synchrophasor data, and the noniterative method is used to compute the distribution voltage level state estimator solution. The distribution systems consist of separate networks of limited size, which can be solved efficiently by the non-iterative method. 


\section{Appendix:}

\section{Variable Product Pair Combinations and Their Relation to Power System Network Topology}

In the non-iterative state estimation method, a list of unique product pairs of the $y$ and $z$ variables satisfying (8) have to be identified. The set of valid product pairs is determined by the system network topology and available measurements, and only the connections of buses and lines with the configurations shown in Figure 4 contribute valid variable pairs. With this knowledge, a search can be conducted through the system topology for the specific connections and generate a list of possible pairs to examine. This appendix lists the equations generated by the two-bus and three-bus connections shown in Figure $4 \mathrm{~A}$ and Figure 4B.

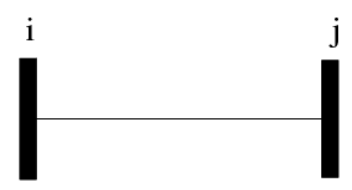

A. Two-Bus

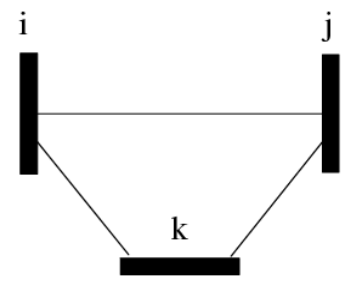

C. Three-Bus Ring

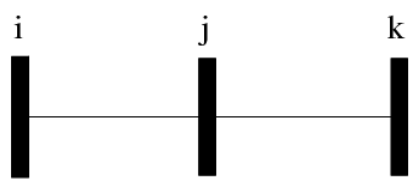

B. Three-Bus

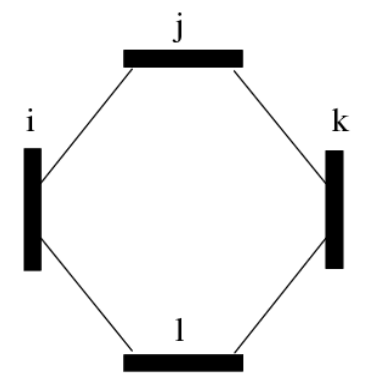

D. Four-Bus Ring

Figure 4 - Bus Connections with Variable Pair Combinations

Two buses, Bus $i$ and Bus $j$ are connected by a line as shown in Figure 4A. If the voltage magnitude measurements on both buses and line power flow measurements from both directions are available, the quadratic voltage variables that would exist in the system are:

$$
\begin{gathered}
V_{j R}^{2}=V_{j R} V_{j R}, \quad V_{j I}^{2}=V_{j I} V_{j I} \\
V_{i R} V_{j R}, V_{i I} V_{j I}, V_{i I} V_{j R}, V_{i R} V_{j I}
\end{gathered}
$$

where the first subscript is the bus index and the second subscript indicates the real or imaginary component.

From these quadratic variables, five valid product pairs can be generated:

$$
\begin{aligned}
\left(V_{i R} V_{i R}\right)\left(V_{j R} V_{j R}\right) & =\left(V_{i R} V_{j R}\right)\left(V_{i R} V_{j R}\right) \\
\left(V_{i R} V_{i R}\right)\left(V_{j I} V_{j I}\right) & =\left(V_{i R} V_{j I}\right)\left(V_{i R} V_{j I}\right) \\
\left(V_{i I} V_{i I}\right)\left(V_{j R} V_{j R}\right) & =\left(V_{i I} V_{j R}\right)\left(V_{i I} V_{j R}\right) \\
\left(V_{i I} V_{i I}\right)\left(V_{j I} V_{j I}\right) & =\left(V_{i I} V_{j I}\right)\left(V_{i I} V_{j I}\right)
\end{aligned}
$$




$$
\left(V_{i R} V_{j R}\right)\left(V_{i I} V_{j I}\right)=\left(V_{i R} V_{j I}\right)\left(V_{i I} V_{j R}\right)
$$

Three buses, $i, j$ and $k$ are connected as shown in Figure Figure 4B. In addition to the two-bus product pairs generated by the $i-j$ and $j-k$ connections, the three-bus connection generates 4 more distinct product pairs:

$$
\begin{aligned}
\left(V_{i R} V_{j R}\right)\left(V_{j I} V_{k R}\right) & =\left(V_{i R} V_{j I}\right)\left(V_{j R} V_{k R}\right) \\
\left(V_{i I} V_{j R}\right)\left(V_{j I} V_{k R}\right) & =\left(V_{i I} V_{j I}\right)\left(V_{j R} V_{k R}\right) \\
\left(V_{i R} V_{j R}\right)\left(V_{j I} V_{k I}\right) & =\left(V_{i R} V_{j I}\right)\left(V_{j R} V_{k I}\right) \\
\left(V_{i I} V_{j R}\right)\left(V_{j I} V_{k I}\right) & =\left(V_{i I} V_{j I}\right)\left(V_{j R} V_{k I}\right)
\end{aligned}
$$

The equations from the other two connections can be found in [7].

\section{Acknowledgment}

This research is supported in part by the New York Power Authority (NYPA) and the New York State Energy Research and Development Authority (NYSERDA), and in part by the Engineering Research Center Program of the National Science Foundation and the Department of Energy under NSF Award Number EEC-1041877 and the CURENT Industry Partnership Program.

\section{References}

[1] F. C. Schweppe, J. Wildes, and D. B. Rom, "Power system static state estimation, part I, II, III," IEEE Trans. Power App. Syst., vol. PAS-89, no. 1, pp. 120-135, Jan. 1970.

[2] A. Abur and A. G. Exposito, Power System State Estimation: Theory and Implementation. New York: Marcel Dekker, 2004.

[3] A. Monticell, State Estimation in Electric Power System: A Generalized Approach. Norwell, MA: Kluwer, 1999.

[4] A. Bjorck, Numerical Methods for Least Squares Problems. Philadelphia, PA: SIAM, 1999.

[5] B. Fardanesh, "Method and systems for power systems analysis: A non-iterative state solver/estimator for power systems operation and control," U.S. Patent 20050160 128, Jan. 31, 2012.

[6] B. Fardanesh, "Direct non-iterative power system state solution and estimation," in Power and Energy Society General Meeting, 2012 IEEE, San Diego, CA, 2012, pp. 1-6.

[7] X. T. Jiang et al., "Power system state estimation using a non-iterative direct state calculation method," presented at CURENT Annu. Site Visit and Industry Conf., Knoxville, TN, 2013.

[8] X. T. Jiang et al., "Improving performance of the non-iterative direct state estimation method," presented at Power and Energy Conf. Illinois, Champaign, IL, 2014.

[9] A. G. Exposito, C. Gomez-Quiles, and A. de la Villa Jaen, "Bilinear power system state estimation," IEEE Trans. Power Syst., vol. 27, no. 1, pp. 493-501, Feb. 2012.

[10]R. A. Jabr and B. C. Pal, "AC network state estimation using linear measurement functions," IET Generation, Transmission and Distribution, vol. 2, no. 1, pp. 1-6, Jan. 2008.

[11]W. Xu et al., "Series load flow: A novel non-iterative load flow method," IEE Proc. Generation, Transmission and Distribution, vol. 2, no. 1, pp. 251-256, May 1998. 
[12]A. A. El-Ela, "A non-iterative and exact linearization load flow technique for circuit contingency effects in power systems," Electric Power Systems Research, vol. 19, no. 3, pp. 213-218, Oct. 1990.

[13]A. Kipnis and A. Shamir, "Cryptanalysis of the HFE public key cryptosystem," in Proc. Crypto '99, Santa Barbara, CA, 1999, pp. 19-30.

[14]Mathworks Inc. (2014, Mar. 7). MATLAB [Online]. Available: http://www.mathworks.com (Retrieved on Mar. 18, 2014).

[15]A. A. El-Keib et al., "Parallel state estimation in power systems," in Twenty-Second Southeastern Symposium on System Theory, Cookeville, TN, 1990, pp. 255-260.

[16]D. M. Falcao, F. F. Wu, and L. Murphy, "Parallel and distributed state estimation," IEEE Trans. on Power Syst., vol. 10, no. 2, pp. 724-730, May 1995.

[17]Mathworks Inc. (2014, Mar. 7). MATLAB Parallel Computing Toolbox [Online]. Available: http://www.mathworks.com/parallel-computing/ (Retrieved on Mar. 19, 2014). 